\title{
Graph Homomorphisms via Vector Colorings
}

\author{
Chris Godsil ${ }^{1}$, David E. Roberson ${ }^{2}$, Brendan Rooney ${ }^{3}$, Robert \\ Sámal ${ }^{4}$, and Antonios Varvitsiotis ${ }^{5,6}$ \\ ${ }^{1}$ Department of Combinatorics \& Optimization, University of Waterloo \\ ${ }^{2}$ Department of Computer Science, University College London \\ ${ }^{3}$ Department of Mathematical Sciences, KAIST \\ ${ }^{4}$ Computer Science Institute, Charles University \\ ${ }^{5}$ Centre for Quantum Technologies, National University of Singapore \\ ${ }^{6}$ School of Physical and Mathematical Sciences, Nanyang Technological University
}

March 29, 2019

\begin{abstract}
In this paper we study the existence of homomorphisms $G \rightarrow H$ using semidefinite programming. Specifically, we use the vector chromatic number of a graph, defined as the smallest real number $t \geq 2$ for which there exists an assignment of unit vectors $i \mapsto p_{i}$ to its vertices such that $\left\langle p_{i}, p_{j}\right\rangle \leq-1 /(t-1)$, when $i \sim j$. Our approach allows to reprove, without using the Erdős-Ko-Rado Theorem, that for $n>2 r$ the Kneser graph $K_{n: r}$ and the $q$-Kneser graph $q K_{n: r}$ are cores, and furthermore, that for $n / r=n^{\prime} / r^{\prime}$ there exists a homomorphism $K_{n: r} \rightarrow K_{n^{\prime}: r^{\prime}}$ if and only if $n$ divides $n^{\prime}$. In terms of new applications, we show that the even-weight component of the distance $k$-graph of the $n$-cube $H_{n, k}$ is a core and also, that non-bipartite Taylor graphs are cores. Additionally, we give a necessary and sufficient condition for the existence of homomorphisms $H_{n, k} \rightarrow H_{n^{\prime}, k^{\prime}}$ when $n / k=n^{\prime} / k^{\prime}$. Lastly, we show that if a 2-walk-regular graph (which is non-bipartite and not complete multipartite) has a unique optimal vector coloring, it is a core. Based on this sufficient condition we conducted a computational study on Ted Spence's list of strongly regular graphs [25] and found that at least $84 \%$ are cores.
\end{abstract}

\section{Introduction}

A homomorphism from a graph $G$ to a graph $H$ is an adjacency preserving map from $V(G)$ to $V(H)$. Formally, a function $\varphi: V(G) \rightarrow V(H)$ is a homomorphism from $G$ to $H$ if $\varphi(u)$ and $\varphi(v)$ are adjacent in $H$ whenever $u$ and $v$ are adjacent in $G$. If there exists a homomorphism from $G$ to $H$ we write $G \rightarrow H$.

Many well-known graph parameters can be defined through graph homomorphisms. One example is the chromatic number of a graph $G$, denoted by $\chi(G)$, which is defined as is the least number of colors required to color the vertices of $G$ such that no two adjacent vertices receive the same color. Equivalently, $\chi(G)$ is the minimum integer $m$ so that $G \rightarrow K_{m}$, where $K_{m}$ is the complete graph on $m$ vertices. Other examples include the clique number, the fractional 
chromatic number, and the circular chromatic number. The interested reader is referred to $[12,15]$ for an extensive survey of graph homomorphisms.

In this work we study the existence of homomorphisms from a graph $G$ to a graph $H$. This problem is important as many important graph-theoretic questions can be phrased as deciding the existence of a homomorphism between two graphs. Nevertheless, it is known that for a non-bipartite graph $H$, deciding whether a graph has a homomorphism to $H$ is NP-hard [13].

In this paper we study the existence of homorphisms $G \rightarrow H$ using semidefinite programming, and more specifically, using vector colorings. For $d \geq 1$ and $t \geq 2$, let $\mathcal{S}_{t}^{d}$ be the infinite graph whose vertices are the unit vectors in $\mathbb{R}^{d}$, where two unit vectors are adjacent if and only if their inner product is at most $-1 /(t-1)$. A homomorphism from $G$ to $\mathcal{S}_{t}^{d}$ is called a vector $t$-coloring of $G$. Equivalently, a vector $t$-coloring of $G$ is an assignment $i \mapsto p_{i}$ of unit vectors in $\mathbb{R}^{d}$ to the vertices of $G$ such that

$$
\left\langle p_{i}, p_{j}\right\rangle \leq \frac{-1}{t-1} \quad \text { whenever } \quad i \sim j,
$$

where $\langle\cdot, \cdot\rangle$ denotes the standard inner product in $\mathbb{R}^{d}$. Note that we will often assume that the vertex set of a graph is $[n]=\{1, \ldots, n\}$ unless otherwise specified.

The vector chromatic number of $G$ is the smallest $t \geq 2$ for which $G \rightarrow \mathcal{S}_{t}^{d}$ (for some integer $d \geq 1$ ) and is denoted $\chi_{v}(G)$. By convention, the vector chromatic number of the empty graph is equal to one. We call a vector $t$ coloring of $G$ optimal if $t=\chi_{v}(G)$. Note that without loss of generality we can always set $d=|V(G)|$, as the space spanned by (the images of) the vertices of $G$ has dimension at most $|V(G)|$. A vector $t$-coloring is strict if every inequality in (1) is met with equality. The strict vector chromatic number of $G$, denoted $\chi_{s v}(G)$, is the smallest $t \geq 2$ for which $G$ has a strict vector $t$-coloring.

Both $\chi_{v}(G)$ and $\chi_{s v}(G)$ were originally introduced by Karger et al. [16] as relaxations of $\chi(G)$. These parameters satisfy the relation $\chi_{v}(G) \leq \chi_{s v}(G) \leq \chi(G)$. Karger et al. noted that $\chi_{s v}(G)$ is the Lovász theta function of the complement of $G$ [16]. Furthermore, $\chi_{v}(G)=\vartheta^{\prime}(\bar{G})$ where $\vartheta^{\prime}$ is a variant of the Lovász theta introduced in [24] and [20].

We study the existence of homorphisms $G \rightarrow H$ when the graphs $G$ and $H$ have the same vector chromatic number, i.e., $\chi_{v}(H)=\chi_{v}(G)$. The highlevel idea is the following. If $\varphi_{1}$ is an optimal vector coloring of $H$ and $\varphi_{2}$ a homomorphism from $G$ to $H$, the map $\varphi_{1} \circ \varphi_{2}$ is an optimal vector coloring of $G$. As a consequence, properties of optimal vector colorings of $G$ translate to properties of homomorphisms $G \rightarrow H$. As an example, if all optimal vector colorings of $G$ are injective, any homomorphism $G \rightarrow H$ will also be injective.

Since the vector chromatic number of a graph is given by a semidefinite program, an optimal vector coloring can be identified to arbitrary precision in polynomial time. Nevertheless, finding the set of all optimal vector colorings is in general a hard problem. For this reason, in this paper we further restrict our attention to graphs that are uniquely vector colorable (UVC), i.e., any two optimal vector colorings differ only by an orthogonal transformation.

Formally, a graph $G$ is called uniquely (strict) vector colorable if for any two optimal (strict) vector colorings $i \mapsto p_{i} \in \mathbb{R}^{d}$ and $i \mapsto q_{i} \in \mathbb{R}^{d^{\prime}}$ the corresponding Gram matrices coincide, i.e.,

$$
\operatorname{Gram}\left(p_{1}, \ldots, p_{n}\right)=\operatorname{Gram}\left(q_{1}, \ldots, q_{n}\right) .
$$


We say that $i \mapsto p_{i}$ is the unique optimal vector coloring of $G$ if for any other optimal vector coloring $i \mapsto q_{i}$, Equation (2) holds. Furthermore, we say that two vector colorings $i \mapsto p_{i}$ and $i \mapsto q_{i}$ are congruent if they satisfy (2).

Although deciding whether a graph is UVC is hard, there exist sufficient conditions for showing that a graph is UVC. For example, such conditions were developed in [8] where it was shown that the Kneser graphs $K_{n: r}$ and their $q$-analogs, the $q$-Kneser graphs $q K_{n: r}$ are UVC. These graphs have nontrivial structure: the vertex set of $K_{n: r}$ consists of the $r$-subsets of [n], with disjoint subsets being adjacent. Similarly, the vertices of $q K_{n: r}$ are the $r$-dimensional subspaces of $\mathbb{F}_{q}^{n}$, two being adjacent if they intersect in the trivial subspace. Furthermore, UVC graphs are interesting in their own right. They were first introduced in [22] to construct tractable instances of the graph realization problem. In the same work UVC graphs were used to construct uniquely colorable graphs. UVC graphs are also closely related to the notion of universal completability (equivalently, the universal rigidity of apex graphs). This in turn is relevant to the low-rank matrix completion problem [18].

\subsection{Summary of results and paper organization}

Graph endomorphisms. In Section 3 we study the existence of endomorphisms of a graph $G$, i.e., homomorphisms from $G$ to itself. Our goal is to find sufficient conditions to show that $G$ does not admit any endomorphisms to a proper subgraph. Graphs that have this property are known as cores.

For an arbitrary graph $G$, the core of $G$ is the vertex minimal subgraph to which $G$ admits a homomorphism. Every graph has a unique core, and the core of $G$ is itself a core. Moreover, $G$ and $H$ have the same core if and only if they are homomorphically equivalent, i.e., $G \rightarrow H$ and $H \rightarrow G$. Cores are the unique minimal elements of these homomorphic equivalence classes. In this sense, the core of a graph is the smallest graph retaining all its homomorphic information.

It is known that deciding whether a graph is a core is a co-NP-complete problem [14]. In Section 3.1 we show that if $G$ is UVC, and its unique optimal vector coloring is injective on the neighborhood of each vertex, then $G$ is a core.

To illustrate the usefulness of this sufficient condition, in Section 3.2 we show that for $n \geq 2 r+1$, both the Kneser graph $K_{n: r}$ and the $q$-Kneser graph $q K_{n: r}$ are cores. Although this is well-known [12], our proof avoids invoking the ErdősKo-Rado Theorem, used to describe the structure of the maximum independent sets of these graphs, and it also avoids using the No Homomorphism Lemma [10].

In terms of new applications, we show that a family of Hamming distance graphs constructed from the $k$-distance graphs of the $n$-cube are cores. These graphs, denoted $H_{n, k}$, have the even weight binary strings of length $n$ as their vertices, two being adjacent if they differ in precisely $k$ positions. In Section 3.3, we show that these graphs are UVC for even $k \in[n / 2+1, n-1]$. In Section 3.4 we focus on 2-walk-regular graphs. We show that if a 2-walk-regular graph (that is not bipartite or complete multipartite) is UVC, it is also a core. Furthermore, in Section 3.5 we show that non-bipartite Taylor graphs are cores.

Finally, in Section 3.6 we give an algorithm for testing whether a 2-walkregular graph is a core. We apply this algorithm to 73816 strongly regular graphs obtained from Ted Spence's webpage [25], showing that 62168 (approx. 84\%) of them are UVC, and therefore cores. 
Homomorphisms between graphs with $\chi_{v}(G)=\chi_{v}(H)$. In Section 4, we study necessary and sufficient conditions for the existence of homomorphisms from $G$ to $H$ for a pair of graphs satisfying $\chi_{v}(G)=\chi_{v}(H)$.

In Section 4.1 we focus on Kneser graphs. It is an open problem to determine all possible homomorphisms between Kneser graphs (e.g., see [7, Problem 11.2]). On the positive side, using the Erdös-Ko-Rado Theorem, Stahl showed in [26] that if $n / r=n^{\prime} / r^{\prime}$, then $K_{n: r} \rightarrow K_{n^{\prime}: r^{\prime}}$ if and only if $n^{\prime}$ is an integer multiple of $n$. As the condition $n / r=n^{\prime} / r^{\prime}$ is equivalent to $\chi_{v}\left(K_{n: r}\right)=\chi_{v}\left(K_{n^{\prime}: r^{\prime}}\right)$, we are able to reprove this result using our approach.

In Section 4.2 we consider the family of $q$-Kneser graphs. Again, we study the existence of homomorphisms from $q K_{n: r}$ to $q^{\prime} K_{n^{\prime}: r^{\prime}}$ where $\chi_{v}\left(q K_{n: r}\right)=$ $\chi_{v}\left(q^{\prime} K_{n^{\prime}: r^{\prime}}\right)$. Our main result is that, under this assumption, the existence of a homomorphism from $q K_{n: r}$ to $q^{\prime} K_{n^{\prime}: r^{\prime}}$ implies that the $q$-binomial coefficient $\left[n^{\prime}\right]_{q}$ is an integer multiple of the $q^{\prime}$-binomial coefficient $[n]_{q^{\prime}}$.

Finally, in Section 4.3 we give necessary and sufficient conditions for the existence of homomorphisms $H_{n, k} \rightarrow H_{n^{\prime}, k^{\prime}}$ when $\chi_{v}\left(H_{n, k}\right)=\chi_{v}\left(H_{n^{\prime}, k^{\prime}}\right)$.

\section{Preliminaries}

\subsection{Basic definitions and notation}

Throughout we set $[n]=\{1, \ldots, n\}$. We denote by $e_{i}$ the $i^{\text {th }}$ standard basis vector, by $\mathbf{1}$ the all-ones vector and by $\mathbf{0}$ the all-zeros vector of appropriate size. All vectors are column vectors. We denote by $\langle\cdot, \cdot\rangle$ the usual inner product between two real vectors. Furthermore, we denote by $\operatorname{span}\left(p_{1}, \ldots, p_{n}\right)$ the linear span of the vectors $\left\{p_{i}\right\}_{i=1}^{n}$. The set of $n \times n$ real symmetric matrices is denoted by $\mathcal{S}^{n}$, and the set of matrices in $\mathcal{S}^{n}$ with nonnegative eigenvalues, i.e., the real positive semidefinite matrices, is denoted by $\mathcal{S}_{+}^{n}$. Given a matrix $X \in \mathcal{S}^{n}$ we denote its kernel/null space by $\operatorname{Ker} X$ and its image/column space by $\operatorname{Im} X$. The Schur product of two matrices $X, Y \in \mathcal{S}^{n}$, denoted by $X \circ Y$, is the matrix whose entries are given by $(X \circ Y)_{i j}=X_{i j} Y_{i j}$ for all $i, j \in[n]$. A matrix $X \in \mathcal{S}^{n}$ has real eigenvalues, and we denote the smallest one by $\lambda_{\min }(X)$. The Gram matrix of a set of vectors $v_{1}, \ldots, v_{n}$, denoted by $\operatorname{Gram}\left(v_{1}, \ldots, v_{n}\right)$, is the $n \times n$ matrix with $i j$-entry equal to $\left\langle v_{i}, v_{j}\right\rangle$. The matrix $\operatorname{Gram}\left(v_{1}, \ldots, v_{n}\right)$ is positive semidefinite and its rank is equal to the dimension of $\operatorname{span}\left(p_{1}, \ldots, p_{n}\right)$. We denote by $\operatorname{sum}(X)$ the sum of all entries in $X$ and use that $\operatorname{sum}(X \circ Y)=\operatorname{Tr}\left(X Y^{T}\right)$.

\subsection{1-walk-regular graphs}

A graph $G$ with adjacency matrix $A$ is said to be 1 -walk-regular if for all $k \in \mathbb{N}$, there exist constants $a_{k}$ and $b_{k}$ such that

(i) $A^{k} \circ I=a_{k} I$;

(ii) $A^{k} \circ A=b_{k} A$.

Equivalently, a graph is 1-walk-regular if for all $k \in \mathbb{N},(i)$ the number of walks of length $k$ starting and ending at a vertex does not depend on the choice of vertex, and (ii) the number of walks of length $k$ between the endpoints of an edge does not depend on the edge. 
Note that a 1-walk-regular graph must be regular. Also, any graph which is vertex and edge transitive is easily seen to be 1-walk-regular. More generally, any graph which is a single class of an association scheme is 1-walk-regular. These include distance regular and, more specifically, strongly regular graphs, the latter of which is the focus of Section 3.6.

Graphs that are 1-walk-regular are particularly relevant to this work because they have a canonical vector coloring and furthermore, there exists a necessary and sufficient condition for this to be the unique vector coloring of such a graph. We first give the definition of the canonical vector coloring.

Definition 2.1. Consider a 1-walk-regular graph $G=([n], E)$ and let $d$ be the multiplicity of the least eigenvalue of its adjacency matrix. Furthermore, let $Q$ be an $n \times d$ matrix whose columns form an orthonormal basis for the eigenspace of the least eigenvalue of $G$ and let $p_{i} \in \mathbb{R}^{d}$ be the $i$-th row of $Q$. The assignment $i \mapsto \sqrt{\frac{n}{d}} p_{i} \in \mathbb{R}^{d}$ is a vector coloring of $G$ which we call the canonical vector coloring.

Consider a 1-walk-regular graph $G$ with least eigenvalue $\tau$. Note that the vectors in the canonical vector coloring linearly span the ambient space, i.e., $\operatorname{span}\left(p_{1}, \ldots, p_{n}\right)=\mathbb{R}^{d}$. Also, the canonical vector coloring of $G$ is not uniquely defined since there are many choices of orthonormal basis for the least eigenspace. Nevertheless, all canonical vector colorings are congruent and thus indistinguishable for our purposes. Indeed, for any orthonormal basis of the least eigenspace, the Gram matrix of the corresponding canonical vector coloring is equal to a scalar multiple of the orthogonal projector $E_{\tau}$ onto the least eigenspace of $G$. To see this, let $Q$ be the matrix whose columns are the chosen orthonormal basis vectors, and consider how the matrix $Q Q^{\top}$ acts on the least eigenspace of $G$ and its orthogonal complement. Furthermore, it follows by the definition of a canonical vector coloring that $\tau p_{i}=\sum_{j \sim i} p_{j}$ for all $i \in V(G)$.

Lastly, recall that the projector $E_{\tau}$ onto the least eigenspace of a graph $G$ is a polynomial in the adjacency matrix of $G$. Concretely, we have that $E_{\tau}=\prod_{\lambda \neq \tau} \frac{1}{\tau-\lambda}(A-\lambda I)$. Thus, if $G$ is 1-walk-regular, the diagonal entries of $E_{\tau}$ and the entries of $E_{\tau}$ that correspond to edges of $G$ are constant.

We are now ready to give a necessary and sufficient condition for a 1-walkregular graph to be UVC.

Theorem $2.2([8])$. Let $G=([n], E)$ be 1-walk-regular with degree $k$ and let $i \mapsto p_{i} \in \mathbb{R}^{d}$ be its canonical vector coloring. Then, we have that:

(i) $\chi_{v}(G)=1-\frac{k}{\lambda_{\min }(G)}$ and $i \mapsto p_{i}$ is an optimal strict vector coloring of $G$.

(ii) $G$ is uniquely vector colorable if and only if for any $R \in \mathcal{S}^{d}$ we have

$$
p_{i}^{\top} R p_{j}=0 \text { for all } i \simeq j \Longrightarrow R=0 .
$$

where $i \simeq j$ means that the vertices $i$ and $j$ are either equal or adjacent.

We note that the calculation for the vector chromatic number of a 1-walkregular graph was first done in [9, Lemma 5.2]. 


\section{Graph cores}

\subsection{A sufficient condition for a graph to be a core}

A homomorphism $\varphi$ is locally injective if it acts injectively on the neighborhood of any vertex, i.e., if $\varphi(u) \neq \varphi(v)$ for any two vertices $u$ and $v$ that have a common neighbor. We recall the following property of endomorphisms proved by Nešetril which we use to make the connection between cores and vector colorings:

Theorem 3.1 ([21]). Let $G$ be a connected graph. Every locally injective endomorphism of $G$ is an automorphism.

This allows us to prove the following simple lemma which is essential to our results on cores.

Lemma 3.2. If $G$ is a connected graph, then $G$ is a core if and only if there exists a (possibly infinite) graph $H$ such that $G \rightarrow H$ and every homomorphism from $G$ to $H$ is locally injective.

Proof. If $G$ is a core, then set $H=G$ and we are done. Conversely, suppose $G$ is connected and not a core. Further suppose that $G \rightarrow H$. We will show that there exists a homomorphism from $G$ to $H$ that is not locally injective. Since $G$ is not a core, there exists an endomorphism $\rho$ of $G$ which is not an automorphism. By Lemma $3.1 \rho$ is not locally injective. Let $\varphi$ be any homomorphism from $G$ to $H$. It is easy to see that $\varphi \circ \rho$ is a homomorphism from $G$ to $H$ that is not locally injective.

We can apply the above in the case of $H=\mathcal{S}_{t}^{d}$ to obtain our main result relating vector colorings to cores, presented as Theorem 3.3 below. Note that a vector coloring is injective (resp. locally injective) if it is injective (resp. locally injective) as a homomorphism to $\mathcal{S}_{t}^{d}$ for some $d \in \mathbb{N}$ and $t \geq 2$. Equivalently, a vector coloring is (locally) injective if it does not map any two vertices (at distance two from each other) to the same vector.

Theorem 3.3. Let $G$ be a connected graph. If every optimal (strict) vector coloring of $G$ is locally injective, then $G$ is a core. In particular, if $G$ is $U V C$ and its unique vector coloring is locally injective, then $G$ must be a core.

We note that in practice, local injectivity does not seem to be a strong restriction. In fact all of the vector colorings discussed in this paper are injective. Furthermore, in Section 3.4 we study a class of graphs which always have locally injective vector colorings.

\subsection{Kneser graphs are cores}

Using Theorem 3.3 combined with our results on unique vector colorability we now proceed to show that several graph families are cores.

Corollary 3.4. For $n \geq 2 r+1$, the graphs $K_{n: r}$ and $q K_{n: r}$ are cores.

Proof. It was shown in [8] that for $n \geq 2 r+1$, both $K_{n: r}$ and $q K_{n: r}$ are UVC. Moreover, their canonical vector colorings are injective (e.g., see (18) and (20) in the Appendix). The proof is concluded using Theorem 3.3. 
As already mentioned in the introduction this result is well-known, e.g. see [12]. Nevertheless, our proof is of independent interest as it does not rely Erdős-Ko-Rado Theorem or the No Homomorphism Lemma.

The above corollary leaves open the case of the $q$-Kneser graphs $q K_{2 r: r}$. In the case where $r=2$, these graphs are transitive on non-edges, and one can use this to show that they are cores. On the other hand, we have shown computationally that $2 K_{4: 2}$ is not UVC. We conjecture that the graphs $q K_{2 r: r}$ are cores but are not UVC, however we have not been able to prove either claim.

\subsection{Hamming graphs are cores}

Consider an abelian group $\Gamma$ and inverse closed connection set $C \subseteq \Gamma \backslash\{0\}$. The Cayley graph corresponding to $\Gamma$ and $C$, denoted by Cay $(\Gamma, C)$, has as its vertex set the elements of $\Gamma$ and two vertices $a, b \in \Gamma$ are adjacent if $a-b \in C$.

In this section we focus on Cayley graphs over $\mathbb{Z}_{2}^{n}$ with group operation bitwise XOR. We refer to the number of 1's in an element of $\mathbb{Z}_{2}^{n}$ as its weight. As a connection set we take all elements of weight $k$, for some fixed $k \in[n]$, which we denote by $C_{n, k}$. Note that the graphs $\operatorname{Cay}\left(\mathbb{Z}_{2}^{n}, C_{n, k}\right)$ lie in the binary Hamming scheme, specifically they are the distance $k$-graphs of the $n$-cube. Furthermore, note that $\operatorname{Cay}\left(\mathbb{Z}_{2}^{n}, C_{n, k}\right)$ is bipartite if $k$ is odd. Also, if $k \neq n$ and $k$ is even, this is a non-bipartite graph with two isomorphic components corresponding to the even and odd weight elements. We denote the component consisting of the even-weight vertices by $H_{n, k}$.

Our main result in this section is that $H_{n, k}$ is UVC for any even integer $k \in[n / 2+1, n-1]$. Note that $H_{n, k}$ is arc transitive, i.e., any ordered pair of adjacent vertices can be mapped to any other such pair by an automorphism of $H_{n, k}$. Therefore, $H_{n, k}$ is 1-walk-regular and thus we can use Theorem 2.2 to show it is UVC. For this, we need to determine the canonical vector coloring of $H_{n, k}$ and show that condition (3) is satisfied.

As a first step we calculate the least eigenvalue of $H_{n, k}$. As $\operatorname{Cay}\left(\mathbb{Z}_{2}^{n}, C_{n, k}\right)$ consists of two-isomorphic connected components, the spectrum of $H_{n, k}$ coincides with the spectrum of $\operatorname{Cay}\left(\mathbb{Z}_{2}^{n}, C_{n, k}\right)$ which can be calculated as follows: The eigenvectors of a Cayley graph for an abelian group can be constructed using the characters of the underlying group. In particular, if $\chi$ is a character of $\Gamma$, then the vector $(\chi(a))_{a \in \Gamma}$ is an eigenvector for $\operatorname{Cay}(\Gamma, C)$ with eigenvalue $\sum_{c \in C} \chi(c)$. Moreover, ranging over all $|\Gamma|$ characters we get a full orthogonal set of eigenvectors. For details on the spectra of Cayley graphs see [19] or [1].

Recall that the characters of $\mathbb{Z}_{2}^{n}$ are given by the functions $\chi_{a}(x)=(-1)^{a \cdot x}$, for all $a \in \mathbb{Z}_{2}^{n}$. Throughout, for $x, y \in \mathbb{Z}_{2}^{n}$, we denote by $x \cdot y$ the inner product of $x$ and $y$ considered as vectors over $\mathbb{Z}_{2}$. We also define $x^{\perp}$ to be the set $\left\{y \in \mathbb{Z}_{2}^{n}: x \cdot y=0\right\}$. Each character $\chi_{a}$ corresponds to an eigenvector $v_{a} \in \mathbb{Z}_{2}^{n}$ of $\operatorname{Cay}\left(\mathbb{Z}_{2}^{n}, C_{n, k}\right)$ given by

$$
v_{a}(x)=(-1)^{a \cdot x}, \text { for } x \in \mathbb{Z}_{2}^{n},
$$

with corresponding eigenvalue

$$
\sum_{c \in C_{n, k}}(-1)^{a \cdot c}=\left|C_{n, k} \cap a^{\perp}\right|-\left|C_{n, k} \backslash a^{\perp}\right|=\left(\begin{array}{l}
n \\
k
\end{array}\right)-2\left|C_{n, k} \backslash a^{\perp}\right| .
$$


Lastly, note that

$$
\begin{aligned}
& v_{a}(x)=v_{\mathbf{1}+a}(x), \quad \forall x \in V\left(H_{n, k}\right), \text { and } \\
& v_{a}(x)=-v_{\mathbf{1}+a}(x), \quad \forall x \in \mathbb{Z}_{2}^{n} \backslash V\left(H_{n, k}\right) .
\end{aligned}
$$

By (5) we see that the smallest eigenvalue of $\operatorname{Cay}\left(\mathbb{Z}_{2}^{n}, C_{n, k}\right)$ corresponds to the elements $a \in \mathbb{Z}_{n}^{2}$ that maximize $\left|C_{n, k} \backslash a^{\perp}\right|$. Finding the maximum value of $\left|C_{n, k} \backslash a^{\perp}\right|$ was already considered by Engström et al. [6]. They gave and inductive proof of the bound in the theorem below, but we also need to determine when equality is attained in this bound. However, their proof can be easily modified to achieve this: simply include the claim about attainment in their induction hypothesis. Thus we have the following:

Theorem $3.5([6])$. For any even integer $k \in\left[\frac{n+1}{2}, n\right]$ we have that

$$
\left|C_{n, k} \backslash a^{\perp}\right| \leq\left(\begin{array}{l}
n-1 \\
k-1
\end{array}\right), \quad \forall a \in \mathbb{Z}_{2}^{n} .
$$

Moreover, equality is attained in (7) if a has weight 1 or $n-1$. If $k \in$ $\left[\frac{n}{2}+1, n-1\right]$, then these are the only elements where equality is attained.

Based on Theorem 3.5 we now compute the canonical vector coloring of $H_{n, k}$.

Lemma 3.6. For any even integer $k \in\left[\frac{n+1}{2}, n\right]$ we have that

$$
\lambda_{\min }\left(H_{n, k}\right)=\frac{n-2 k}{k}\left(\begin{array}{l}
n-1 \\
k-1
\end{array}\right), \quad \text { and } \quad \chi_{v}\left(H_{n, k}\right)=\frac{2 k}{2 k-n} .
$$

Furthermore, for any even integer $k \in[n / 2+1, n-1]$, the canonical vector coloring of $H_{n, k}$ is given by $x \mapsto p_{x} \in \mathbb{R}^{n}$ where

$$
p_{x}(i)=\frac{(-1)^{x_{i}}}{\sqrt{n}}, \quad \forall i \in[n] .
$$

Proof. As previously noted, the least eigenvalue of $H_{n, k}$ is equal to the least eigenvalue of $\operatorname{Cay}\left(\mathbb{Z}_{2}^{n}, C_{n, k}\right)$. The latter is equal to $\frac{n-2 k}{k}\left(\begin{array}{l}n-1 \\ k-1\end{array}\right)$ by (5) and Theorem 3.5. Furthermore, as $H_{n, k}$ is 1-walk-regular, Theorem $2.2(i)$ implies $\chi_{v}\left(H_{n, k}\right)=\frac{2 k}{2 k-n}$.

Next, consider an even integer $k \in[n / 2+1, n-1]$. By Theorem 3.5, the least eigenvalue of $\operatorname{Cay}\left(\mathbb{Z}_{2}^{n}, C_{n, k}\right)$ has multiplicity $2 n$. In particular, a set of orthogonal eigenvectors is given by $\left\{v_{e_{i}}\right\}_{i=1}^{n} \cup\left\{v_{1+e_{i}}\right\}_{i=1}^{n}$. For all $i \in[n]$ write $v_{e_{i}}$ as $\left(x_{i}, y_{i}\right)^{\mathrm{T}}$ where $x_{i}$ is the restriction of $v_{e_{i}}$ on $V\left(H_{n, k}\right)$ and $y_{i}$ its restriction on $\mathbb{Z}_{2}^{n} \backslash V\left(H_{n, k}\right)$. Using (6) it follows that $v_{\mathbf{1}+e_{i}}=\left(x_{i},-y_{i}\right)$ for all $i \in[n]$. As $\left\langle v_{e_{i}}, v_{e_{j}}\right\rangle=\left\langle v_{e_{i}}, v_{1+e_{j}}\right\rangle=0$, for all $i \neq j$, the vectors $\left\{x_{i}\right\}_{i=1}^{n}$ are pairwise orthogonal. Furthermore, note that the multiplicity of $\lambda_{\min }\left(H_{n, k}\right)$ as an eigenvalue of $H_{n, k}$ is $n$ (because its multiplicity as an eigenvalue of $\operatorname{Cay}\left(\mathbb{Z}_{2}^{n}, C_{n, k}\right)$ is $2 n)$. Thus, the vectors $\left\{\frac{x_{i}}{\sqrt{2^{n-1}}}: i \in[n]\right\}$ form an orthonormal basis of the least eigenspace of $H_{n, k}$. Lastly, according to Definition 2.1, to construct the canonical vector coloring of $H_{n, k}$ we consider the vectors $\left\{\frac{x_{i}}{\sqrt{2^{n-1}}}: i \in[n]\right\}$ as columns of a matrix and then we scale its rows by $\sqrt{\frac{2^{n-1}}{n}}$. This shows that the canonical vector coloring of $H_{n, k}$ is given by (9). 
Lastly, to show that $H_{n, k}$ is UVC, we must show that its canonical vector coloring satisfies (3). This is accomplished in the following lemma.

Lemma 3.7. Let $x \mapsto p_{x} \in \mathbb{R}^{n}$ be the canonical vector coloring of $H_{n, k}$. Then, for any $n \times n$ symmetric matrix $R$ we have that

$$
p_{x}^{\top} R p_{y}=0, \text { for all } x \simeq y \Longrightarrow R=0 .
$$

Proof. Since $\operatorname{span}\left\{p_{x}: x \in V\left(H_{n, k}\right)\right\}=\mathbb{R}^{n}$ we just need to show that $R p_{x}=0$, for all $x \in V\left(H_{n, k}\right)$. For this consider the subspace

$$
V_{x}:=\operatorname{span}\left\{p_{y}: y \simeq x\right\},
$$

and note that the hypothesis of (10) can be equivalently expressed as $R p_{x} \in V_{x}^{\perp}$, for all $x \in V\left(H_{n, k}\right)$. Thus, if we can show that $V_{x}=\mathbb{R}^{n}$, for all $x \in V\left(H_{n, k}\right)$, we get from Equation (10) that $R p_{x}=0$ for all $x \in V\left(H_{n, k}\right)$, and we are done.

We first consider the case of $V_{x}$ when $x=\mathbf{0}$, the vector of all zeros in $\mathbb{Z}_{2}^{n}$. The neighbors of $\mathbf{0}$ are all the vectors of weight $k$ in $\mathbb{Z}_{2}^{n}$. For each pair of distinct $i, j \in[n]$, there exist weight $k$ vectors $y, z \in \mathbb{Z}_{2}^{n}$ such that $e_{i}-e_{j}=\frac{\sqrt{n}}{2}\left(p_{y}-p_{z}\right)$. The vectors $y$ and $z$ can be chosen by picking any two weight $k$ vectors that differ only in positions $i$ and $j$. Therefore, $e_{i}-e_{j} \in V_{\mathbf{0}}$ for all $i, j \in[n]$. Since $\operatorname{span}\left\{e_{i}-e_{j}: i \neq j\right\}=\operatorname{span}(\mathbf{1})^{\perp}$, we have that $\operatorname{span}(\mathbf{1})^{\perp} \subseteq V_{\mathbf{0}}$. Lastly, as $\mathbf{1}=\sqrt{n} p_{\mathbf{0}} \in V_{\mathbf{0}}$ (recall (9)) it follows that $V_{\mathbf{0}}=\mathbb{R}^{n}$.

Next, consider an arbitrary $x \in \mathbb{Z}_{2}^{n}$. Note that $V_{x}=\operatorname{Diag}\left(p_{x}\right) V_{\mathbf{0}}$, where $\operatorname{Diag}\left(p_{x}\right)$ is the diagonal matrix with entries corresponding to $p_{x}$. As $V_{\mathbf{0}}=\mathbb{R}^{n}$ and the matrix $\operatorname{Diag}\left(p_{x}\right)$ is invertible, we have that $V_{x}=\mathbb{R}^{n}$, for all $x \in \mathbb{Z}_{2}^{n}$.

Putting everything together we get:

Theorem 3.8. The graph $H_{n, k}$ is $U V C$ for any even integer $k \in[n / 2+1, n-1]$.

It is worth noting that Theorem 3.8 does not hold for all even values of $k$. It is not difficult to show that for $n=2 k-1$, the weight two elements of $\mathbb{Z}_{2}^{n}$ also give eigenvectors corresponding to the least eigenvalue of $H_{n, k}$. Moreover, one can show that these eigenvectors can be used to construct a different optimal vector coloring of $H_{n, k}$. Therefore $H_{n, k}$ is not uniquely vector colorable for $n=2 k-1$ for any even $k$.

The canonical vector coloring of $H_{n, k}$ given in (9) is injective (so in particular it is locally injective). Combining Theorem 3.8 and Theorem 3.3 we get:

Corollary 3.9. The graph $H_{n, k}$ is a core for any even integer $k \in[n / 2+1, n-1]$.

For even $k<n / 2+1$, the situation is unclear, but a few special cases are settled. For instance, for $k=2$ it is known that $H_{n, k}$ is a core if and only if $n$ is not a power of two. Also, if $k=n / 2$ then the vertices $x$ and $\mathbf{1}+x$ have the same neighborhood and thus $H_{n, k}$ is not a core in this case. We noted above that for $n=2 k-1$ the graph $H_{n, k}$ is never UVC. In this case $H_{n, k}$ may or may not be a core. In particular, by the above argument we see that $H_{n, k}$ is a core when $k=2$ and $n=5$, however by direct computations we have found that the core of $H_{7,4}$ is the complete graph on 8 vertices. 


\subsection{2-walk-regular graphs}

A graph $G$ is said to be 2-walk-regular if it is 1-walk-regular with the additional property that, for all $k \in \mathbb{N}$, the number of walks of length $k$ with initial and final vertices at distance two from each other does not depend on the specific pair of vertices. In this section we show that, with a few simple exceptions, any uniquely vector colorable 2 -walk-regular graph must be a core.

To show this we need to define the distance 2-graph of a graph $G$. This is the graph with vertex set $V(G)$ in which two vertices are adjacent if they are at distance 2 in $G$. We denote this graph as $G_{2}$. Using this notion we can give another definition of 2-walk-regular graphs: a graph $G$ is 2-walk-regular if it is 1walk-regular and there exist numbers $c_{k}$ for all $k \in \mathbb{N}$ such that $A^{k} \circ A_{2}=c_{k} A_{2}$, where $A_{2}$ is the adjacency matrix of $G_{2}$.

The following lemma gives a relationship between a graph and its distance 2 -graph which we need for the main result of this section.

Lemma 3.10. Let $G$ be a connected graph. The components of $G_{2}$ induce independent sets in $G$ if and only if $G$ is bipartite or complete multipartite.

Proof. It is easy to see that if $G$ is a connected bipartite or complete multipartite graph, then the components of $G_{2}$ induce independent sets in $G$.

To see the converse suppose that $G$ is connected, not bipartite and the components of $G_{2}$ induce independent sets in $G$. We show that $G$ must be complete multipartite. Let $D_{1}, \ldots, D_{k}$ be the vertex sets of the components of $G_{2}$. Since these are independent sets in $G$, coloring vertices in $D_{j}$ with color $j$ gives a proper coloring of $G$. Since $G$ is not bipartite, we have that $k \geq 3$.

We show that any shortest path in $G$ only contains two colors and these alternate along the path. Indeed, consider a shortest path in $G$ which contains three or more colors. Note that consecutive vertices receive different colors (as color classes are independent sets) and thus, there exist three consecutive vertices on this path with distinct colors. However this is a contradiction, since the first and last of these three vertices would be at distance two, and must therefore receive the same color (as they lie in the same component of $G_{2}$ ).

This fact has two useful consequences. First, if a vertex has two neighbors of distinct colors, then they must be adjacent. Second, every vertex has a neighbor of every color other than its own. To see this let $v \in V(G)$ and consider another vertex $u$ with a different color. Then the neighbor of $v$ on the shortest path from $v$ to $u$ (this exists as $G$ is connected) has the required property.

Lastly, towards a contradiction suppose that $G$ is not complete multipartite. This implies there must be two vertices $u_{1}$ and $u_{2}$ in different color classes (say colored 1 and 2 respectively) that are not adjacent. Since they are not adjacent, $u_{1}$ and $u_{2}$ must be at distance at least two. However, by the above, any shortest path between them alternates colors and therefore they must be at distance at least three. Furthermore, by considering the fourth vertex on this path, we can assume that $u_{1}$ and $u_{2}$ are at distance exactly three. Therefore, there exist vertices $v_{1}$ and $v_{2}$ such that $u_{1} \sim v_{2} \sim v_{1} \sim u_{2}$ (note that the subscripts of these vertices correspond to their colors). By the above, $v_{2}$ has a neighbor $w$ of color 3. Since $w$ and $u_{1}$ are vertices of different colors in the neighborhood of $v_{2}$, by the previous paragraph $w$ and $u_{1}$ must be adjacent. Similarly, $w$ and $v_{1}$ are adjacent. This leads to a contradiction. 
Using the above lemma, we are able to show that the canonical vector coloring of a 2-walk-regular graph is always locally injective.

Lemma 3.11. Let $G$ be a connected 2-walk-regular graph that is not bipartite or complete multipartite. The canonical vector coloring of $G$ is locally injective.

Proof. Let $i \mapsto p_{i}$ be the canonical vector coloring of $G$. Recall that the Gram matrix of this vector coloring is a scalar multiple of the projection, $E_{\tau}$, onto the eigenspace of $G$ corresponding to its least eigenvalue. Since $E_{\tau}$ is a polynomial in the adjacency matrix of $G$ and $G$ is 2-walk-regular, there exists a real number $c$ such that $E_{\tau} \circ A_{2}=c A_{2}$, where $A_{2}$ is the adjacency matrix of $G_{2}$. Therefore, $\left\langle p_{i}, p_{j}\right\rangle$ is constant for all vertices $i$ and $j$ at distance 2 in $G$.

Suppose that $i \mapsto p_{i}$ is not locally injective. Then there exist $i, j \in V(G)$ that are at distance two in $G$ such that $p_{i}=p_{j}$. This means that $\left\langle p_{i}, p_{j}\right\rangle=1$, and by the argument in the first paragraph this implies that any pair of vertices at distance two are mapped to the same vector. Therefore, the vertices in a single component of $G_{2}$ are all mapped to the same vector. However, by Lemma 3.10 and the assumption, $G_{2}$ has a component which contains a pair of adjacent vertices, and this pair of vertices cannot be mapped to the same vector since their inner product must be negative. This gives a contradiction and proves the theorem.

The following theorem is a direct consequence of Lemma 3.11.

Theorem 3.12. Let $G$ be a 2-walk-regular, non-bipartite, and not complete multipartite graph. If $G$ is uniquely vector colorable, then $G$ is a core.

Note that we do not need to assume that $G$ is connected in Theorem 3.12 since this is implied by unique vector colorability. Examples of 2-walk-regular graphs include 2-arc-transitive graphs, distance regular graphs, and in particular strongly regular graphs, which we focus on in Section 3.6.

\subsection{Taylor graphs}

A connected graph $G$ of diameter $d$ is distance regular if there exist numbers $p_{i j}^{k}$ for $i, j, k=0,1, \ldots, d$ such that for any pair of vertices $u, v$ at distance $k$ from each other, the number of vertices $w$ at distance $i$ from $u$ and distance $j$ from $v$ is equal to $p_{i j}^{k}$. This turns out to be equivalent to the existence of numbers $b_{0}, \ldots, b_{d-1}$ and $c_{1}, \ldots, c_{d}$ such that for any vertices $u, v$ at distance $i$ in $G$, the number of neighbors of $v$ at distance $i+1$ from $u$ is $b_{i}$ and the number of neighbors of $v$ at distance $i-1$ from $u$ is $c_{i}$. The array $\left\{b_{0}, \ldots, b_{d-1} ; c_{1}, \ldots, d_{d}\right\}$ is known as the intersection array of $G$ and it characterizes many of its properties, such as the eigenvalues of $G$ and the numbers $p_{i j}^{k}$ from above. Also note that the number $b_{0}$ is the valency of $G$.

Another useful property of a distance regular graph $G$ is that the span of the adjacency matrices of its distance graphs is equal to the algebra of polynomials of its adjacency matrix $A$. This implies that any polynomial in $A$ is constant on entries corresponding to pairs of vertices at some fixed distance (similar to 1- and 2-walk-regularity, but for any distance), and that the adjacency matrices of its distance graphs are polynomials in $A$. For a detailed account of distance regular graphs we refer the reader to [2]. 
A Taylor graph is a distance regular graph whose intersection array is given by $\{k, \mu, 1 ; 1, \mu, k\}$, thus they have diameter three. Examples of (non-bipartite) Taylor graphs include the icosahedral graph and the Gosset graph. Moreover, given any strongly regular graph $G$ with parameters $(v, k, a, c)$ (see Section 3.6 for definition) where $k=2 c$, one can construct a non-bipartite Taylor graph as follows: Take two copies $G_{1}$ and $G_{2}$ of $G$, and add an edge between a vertex $u$ of $G_{1}$ and vertex $v$ of $G_{2}$ if the corresponding vertices of $G$ were distinct and non-adjacent. Finally, add a vertex adjacent to every vertex of $G_{1}$ and a vertex adjacent to every vertex of $G_{2}$. This will be a Taylor graph on $2 v+2$ vertices with intersection array $\{v, v-k-1,1 ; 1, v-k-1, v\}$.

The parameters of a Taylor graph imply the number of vertices at distance 1,2 , and 3 from a given vertex is $k, k$, and 1 , respectively. Thus a Taylor graph has $2 k+2$ vertices and every vertex has a unique vertex at distance three from it. We refer to such pairs as antipodes. Note that the antipode of a vertex $u$ is adjacent to every vertex at distance two from $u$. We will show that every Taylor graph is UVC and thus a core unless it is bipartite. First, we need to prove the following lemma.

Lemma 3.13. Let $G$ be a Taylor graph. Then, in the canonical vector coloring of $G$, pairs of vertices at distance three are assigned antipodal vectors.

Proof. Let $A$ be the adjacency matrix of $G$ and $A_{3}$ the adjacency matrix of the distance 3-graph of $G$, denoted $G_{3}$. Note that $G_{3}$ is isomorphic to the disjoint union of some number of $K_{2}$ graphs, and therefore has only two eigenvalues: 1 and -1 . Let $E_{\tau}$ be the projection onto the $\tau$-eigenspace of $G$ where $\tau$ is its least eigenvalue. For $u \in V(G)$ let $p_{u}$ be the vector assigned to $u$ in the canonical vector coloring of $G$. Recall that $E_{\tau}$ is a scalar multiple of the Gram matrix of the $p_{u}$. Let $d$ be the dimension of the $\tau$-eigenspace. $\operatorname{Then} \operatorname{Tr}\left(E_{\tau}\right)=d$, since the trace of a projection is equal to its rank. Furthermore, since $G$ is distance regular, all polynomials in $A$ have constant diagonal, and so all of the diagonal entries of $E_{\tau}$ must be equal to $d / n$, where $n$ is the number of vertices of $G$. We will show that the entries of $E_{\tau}$ corresponding to pairs of vertices at distance three are equal to $-d / n$, which will imply that vectors assigned to such pairs in the canonical vector coloring are antipodal.

Since $A_{3}$ is a polynomial in $A$, we have that $A_{3} E_{\tau}=\lambda E_{\tau}$ where $\lambda$ is some eigenvalue of $G_{3}$, i.e., is \pm 1 . We will show that $\lambda=-1$. To do this, it suffices to show that any $\tau$-eigenvector of $G$ is a -1-eigenvector of $G_{3}$. Suppose that $z$ is a $\tau$-eigenvector of $G$. Then $z$ is an eigenvector of $G_{3}$ with eigenvalue \pm 1 , since these are its only eigenvalues. Suppose for contradiction that $z$ is a 1-eigenvector for $G_{3}$. Since $G_{3}$ is a disjoint union of $K_{2}$ 's whose edges are between antipodes of $G$, this implies that $z$ is constant on pairs of antipodes. Furthermore, since $z$ is a $\tau$-eigenvector of $G$, it is orthogonal to the all ones vector since this is a $k$-eigenvector of $G$. Thus the entries of $z$ sum to zero. Now consider any vertex $u \in V(G)$ such that $z_{u} \neq 0$ and let $S=\{v \in V(G): v \simeq u\}$ be the closed neighborhood of $u$. Then there are no pairs of antipodes contained in $S$ and no pairs of antipodes contained in $V(G) \backslash S$, since this is the closed neighborhood of the antipode of $u$. Thus the antipode relation is a bijection between $S$ and $V(G) \backslash S$. Therefore,

$$
0=\sum_{v \in V(G)} z_{v}=\sum_{v \in S} z_{v}+\sum_{v \in V(G) \backslash S} z_{v}=2 \sum_{v \in S} z_{v} .
$$


This implies that $z_{u}+\sum_{v \sim u} z_{v}=0$ and thus $(A z)_{u}=\sum_{v \sim u} z_{v}=-z_{u}$. Therefore, $z$ is a -1 -eigenvector of $G$. But $\tau \neq-1$ since it is well known that the only connected graphs with least eigenvalue equal to -1 are the complete graphs. Thus $z$ cannot be a $\tau$-eigenvector of $G$, a contradiction.

By the above, we have that $A_{3} E_{\tau}=-E_{\tau}$. Let $\operatorname{sum}(M)$ denote the sum of the entries of the matrix $M$, and note that $\operatorname{sum}(M \circ N)=\operatorname{Tr}(M N)$ for any symmetric matrices $M$ and $N$. Thus we have that

$$
\operatorname{sum}\left(A_{3} \circ E_{\tau}\right)=\operatorname{Tr}\left(A_{3} E_{\tau}\right)=\operatorname{Tr}\left(-E_{\tau}\right)=-d .
$$

Since $E_{\tau}$ is a polynomial in $A$ and $G$ is distance regular, the entries of $E_{\tau}$ corresponding to pairs of vertices at distance three are all equal to some constant $\gamma$. The number of such entries is equal to the number of 1's in $A_{3}$ which is twice the number of edges of $G_{3}$. Since $G_{3}$ is the disjoint union of $K_{2}$ 's, this is just $n$, the number of vertices of $G$. Therefore, $n \gamma=\operatorname{sum}\left(A_{3} \circ E_{\tau}\right)=-d$ and thus $\gamma=-d / n$, which is the negative of the diagonal entries of $E_{\tau}$. Thus for vertices $u$ and $v$ at distance three, $\left\langle p_{u}, p_{v}\right\rangle=-\left\langle p_{u}, p_{u}\right\rangle=-1$, and this implies that $p_{u}=-p_{v}$.

Using the above lemma, we can show that every Taylor graph is UVC.

Theorem 3.14. Any Taylor graph is uniquely vector colorable. Furthermore, this implies that any non-bipartite Taylor graph is a core.

Proof. Let $G$ be a Taylor graph and let $u \mapsto p_{u} \in \mathbb{R}^{d}=\operatorname{span}\left\{p_{v}: v \in V(G)\right\}$ be its canonical vector coloring. In order to prove that $G$ is UVC, we must show that the only symmetric matrix $R$ satisfying $p_{u}^{\top} R p_{v}=0$ for $u \simeq v$ is the zero matrix. Consider the subspace

$$
V_{u}=\operatorname{span}\left\{p_{w}: w \simeq u\right\} .
$$

We will show that $V_{u}=\mathbb{R}^{d}$. Let $v$ be the antipode of $u$. Consider a vertex $x \in V(G)$ whose antipode is $y \in V(G)$. If $x \simeq u$, then $p_{x} \in V_{u}$ by definition, and we are done. Otherwise we must have $y \simeq u$, since $V(G) \backslash\{w \in V(G)$ : $w \simeq u\}=\{w \in V(G): w \simeq v\}$ and it is not possible for both $x$ and $y$ to be contained in the closed neighborhood of $v$ because they are at distance three. If $y \simeq u$ then $-p_{x}=p_{y} \in V_{u}$ and thus $p_{x} \in V_{u}$. Thus $p_{x} \in V_{u}$ for all $x \in V(G)$ and therefore $V_{u}=\mathbb{R}^{d}$, and this holds for all $u \in V(G)$.

The equation $p_{v}^{\top} R p_{u}=0$ for $u \simeq v$ implies that for fixed $u$ the vector $R p_{u}$ lies in $V_{u}^{\perp}$. By the above, this means that $R p_{u}=0$ for all $u \in V(G)$, and thus $R=0$ as desired. This implies that any Taylor graph $G$ is UVC, and thus by Theorem 3.12, $G$ is a core unless it is bipartite or complete multipartite. Since complete multipartite graphs have diameter two, they are never Taylor graphs. Thus we have shown that a Taylor graph is a core unless it is bipartite (which is possible).

We remark that bipartite Taylor graphs are known as crown graphs, i.e., complete bipartite graphs with a perfect matching removed. In terms of the parameters of a Taylor graph this occurs whenever $\mu=k-1$.

\subsection{Computations for strongly regular graphs}

Motivated by Theorem 3.12, we now give an algorithm for showing that a 2walk-regular graph is UVC, and thus, a core. This relies on the following result. 
Lemma 3.15. Let $G$ be a 1-walk-regular graph and let $i \mapsto p_{i} \in \mathbb{R}^{d}$ be its canonical vector coloring. Also, let d be the multiplicity of the least eigenvalue of $G$. Then, $G$ is $U V C$ if and only if

$$
\operatorname{dim}\left(\operatorname{span}\left\{p_{e}: e \in E(G)\right\}\right)=\left(\begin{array}{c}
d+1 \\
2
\end{array}\right),
$$

where

$$
p_{e}:=p_{i} p_{j}^{\top}+p_{j} p_{i}^{\top}, \quad \text { for all } e=\{i, j\} \in E(G),
$$

If $G$ is additionally 2-walk-regular then it is a core unless it is bipartite or complete multipartite.

Proof. Let $G$ be a 2-walk-regular graph, and let $i \mapsto p_{i} \in \mathbb{R}^{d}$ be its canonical vector coloring. Also, recall that the canonical vector coloring satisfies

$$
\tau p_{i}=\sum_{j \sim i} p_{j}, \quad \forall i \in[n],
$$

where $\tau$ is the least eigenvalue of $G$ (which is not zero). Thus, if $p_{i}^{\top} R p_{j}=0$ for all $i \sim j$, it follows by (13) that $p_{i}^{\top} R p_{i}=0$ for all $i \in[n]$.

By Theorem 2.2, the graph $G$ is UVC if and only if condition (3) holds, which by the previous discussion can be equivalently expressed as

$$
p_{i}^{\top} R p_{j}=\frac{\operatorname{Tr}\left(R\left(p_{j} p_{i}^{\top}+p_{i} p_{j}^{\top}\right)\right)}{2}=0, \text { for all } i \sim j \Longrightarrow R=0,
$$

In turn, Equation (14) expresses that the matrices $\left\{p_{e}: e \in E(G)\right\}$ span the space of symmetric $d \times d$ matrices, which has dimension $\left(\begin{array}{c}d+1 \\ 2\end{array}\right)$. The proof is concluded by Theorem 3.12 .

To use Lemma 3.15 we need to determine the canonical vector coloring of $G$ and then compute the matrices $p_{e}$. This requires us to compute an orthonormal basis of the least eigenspace of $G$. However, these eigenvectors may contain irrational entries. Since we are interested in the dimension of the span of the $p_{e}$, our computations must be exact, rather than numerical. Thus, this approach may produce some computational difficulties. Instead, we use a method of determining $\operatorname{dim}\left(\operatorname{span}\left\{p_{e}: e \in E(G)\right\}\right)$ that avoids eigenvector computations. The details of the implementation are given in Appendix B.

As a case study, we applied this method to investigate how often a strongly regular graph happens to be a core. The parameter set of a strongly regular graph (SRG) is a 4-tuple $(v, k, a, c)$ where $v$ is the number of vertices, $k$ is the degree of each vertex, $a$ is the number of common neighbors for every pair of adjacent vertices, and $c$ is the number of common neighbors for each pair of non-adjacent vertices. SRGs are examples of 2-walk-regular graphs that are of significant interest to graph theorists.

The characterization of the cores of SRGs is the subject of a conjecture of Cameron and Kazinidis [3] that was recently verified by Roberson [23]: The core of any strongly regular graph is either itself or a complete graph.

For our data set, we used Ted Spence's list of SRGs available online [25]. The data from these computations is summarized in Table 1 in Appendix B. Overall, approximately $84 \%$ of the strongly regular graphs we tested were UVC 
and therefore cores. A natural question is how many of the non-UVC graphs are cores. By the result of Roberson [23], a SRG is a core if and only if its clique number is not equal to its chromatic number. Using this we verified that only 79 of the 73816 strongly regular graphs we considered are not cores. This shows that almost $99.9 \%$ of all considered instances were cores.

\section{Homomorphisms of graphs with $\chi_{v}(G)=\chi_{v}(H)$}

In this section we give necessary and sufficient conditions for the existence of homomorphisms between two graphs with equal vector chromatic numbers. Our main tool is the following result.

Lemma 4.1. Consider two graphs $G$ and $H$ where $G \rightarrow H, G$ is $U V C$ and $\chi_{v}(G)=\chi_{v}(H)$. If $\varphi_{1}$ is an optimal vector coloring of $H$ and $\varphi_{2}$ is the unique optimal vector coloring of $G$, we have that

$$
\left\{\left\langle\varphi_{2}(g), \varphi_{2}\left(g^{\prime}\right)\right\rangle: g, g^{\prime} \in V(G)\right\} \subseteq\left\{\left\langle\varphi_{1}(h), \varphi_{1}\left(h^{\prime}\right)\right\rangle: h, h^{\prime} \in V(H)\right\} .
$$

Proof. Let $\varphi$ be a homomorphism $G \rightarrow H$. Since $\chi_{v}(G)=\chi_{v}(H)$, the map $\varphi_{1} \circ \varphi$ is an optimal vector coloring of $G$. Lastly, as $G$ is UVC we have that

$$
\left.\left\langle\varphi_{2}(g), \varphi_{2}\left(g^{\prime}\right)\right\rangle: g, g^{\prime} \in V(G)\right\}=\left\{\left\langle\left(\varphi_{1} \circ \varphi\right)(g),\left(\varphi_{1} \circ \varphi\right)\left(g^{\prime}\right)\right\rangle: g, g^{\prime} \in V(G)\right\},
$$

and the latter set is clearly contained in $\left\{\left\langle\varphi_{1}(h), \varphi_{1}\left(h^{\prime}\right)\right\rangle: h, h^{\prime} \in V(H)\right\}$.

As we now show, this simple observation yields some algebraic conditions between $G$ and $H$ which allows us to restrict the possible homomorphisms $G \rightarrow H$.

\subsection{Kneser graphs}

As already mentioned in the introduction, Stahl used the Erdős-Ko-Rado Theorem to show that if $n / r=n^{\prime} / r^{\prime}$, then $K_{n: r} \rightarrow K_{n^{\prime}: r^{\prime}}$ if and only if $n^{\prime}$ is an integer multiple of $n$ (in which case $r^{\prime}$ is an integer multiple of $r$ as well) [26]. Since $\chi_{v}\left(K_{n: r}\right)=n / r$, we can apply Lemma 4.1 to obtain an alternative proof of this result.

Theorem $4.2([26])$. Let $n, r, n^{\prime}, r^{\prime}$ be integers satisfying $n>2 r$ and $n / r=$ $n^{\prime} / r^{\prime}$. Then there exists a homomorphism from $K_{n: r}$ to $K_{n^{\prime}: r^{\prime}}$ if and only if $n^{\prime}$ and $r^{\prime}$ are integer multiples of $n$ and $r$ respectively.

Proof. If $n^{\prime}=m n$ we have that $r^{\prime}=m r$ (as $\left.n / r=n^{\prime} / r^{\prime}\right)$. To show that $K_{n: r} \rightarrow K_{n^{\prime}: r^{\prime}}$ we consider the vertex set of $K_{n^{\prime}: r^{\prime}}$ to be the $r^{\prime}$-subsets of $[m] \times[n]$. The desired homomorphism maps any $r$-subset $S \subseteq[n]$ to $[m] \times S$.

Conversely, consider a homomorphism $\varphi: K_{n: r} \rightarrow K_{n^{\prime}: r^{\prime}}$. By assumption $\gamma:=n / r=n^{\prime} / r^{\prime}$ and thus these two graphs have the same vector chromatic numbers. Given two sets $S, S^{\prime} \subseteq[n]$ with $\left|S \cap S^{\prime}\right|=k$ it follows by (19) that

$$
\left\langle p_{S}, p_{S^{\prime}}\right\rangle=\frac{k}{r} \cdot \frac{\gamma}{\gamma-1}-\frac{1}{\gamma-1},
$$

where $S \mapsto p_{S}$ is the canonical vector coloring of $K_{n: r}$. By Lemma 4.1 we have

$$
\left\{\frac{k}{r} \cdot \frac{\gamma}{\gamma-1}-\frac{1}{\gamma-1}: k \in[r]\right\} \subseteq\left\{\frac{k^{\prime}}{r^{\prime}} \cdot \frac{\gamma}{\gamma-1}-\frac{1}{\gamma-1}: k^{\prime} \in\left[r^{\prime}\right]\right\} .
$$


In particular, it follows by (16) that for $k=1$ there exists a $k^{\prime} \in\left[r^{\prime}\right]$ such that

$$
\frac{1}{r} \cdot \frac{\gamma}{\gamma-1}-\frac{1}{\gamma-1}=\frac{k^{\prime}}{r^{\prime}} \cdot \frac{\gamma}{\gamma-1}-\frac{1}{\gamma-1} .
$$

This holds if and only if $1 / r=k^{\prime} / r^{\prime}$ which is equivalent to $r^{\prime}=k^{\prime} r$. Therefore $r^{\prime}$ is an integer multiple of $r$, and thus $n^{\prime}$ is an integer multiple of $n$.

\section{$4.2 q$-Kneser graphs}

In this section we give a necessary condition for the existence of homomorphisms between $q$-Kneser graphs. Since $\chi_{v}\left(q K_{n: r}\right)=[n]_{q} /[r]_{q}$ we can again use Lemma 4.1. In fact our necessary condition is completely analogous to Theorem 4.2. The only change one needs to make is to replace $n, r$, and $k$ with their $q$-analogues $[n]_{q},[r]_{q}$, and $[k]_{q}$ respectively, noting also that $[1]_{q}=1$.

Theorem 4.3. Let $n, r, q, n^{\prime}, r^{\prime}, q^{\prime}$ be integers satisfying $n>2 r, n^{\prime}>2 r^{\prime}$, and $[n]_{q} /[r]_{q}=\left[n^{\prime}\right]_{q^{\prime}} /\left[r^{\prime}\right]_{q^{\prime}}$. If $q K_{n: r} \rightarrow q^{\prime} K_{n^{\prime}: r^{\prime}}$, then

$$
\left\{\frac{[k]_{q}}{[r]_{q}}: k \in[r]\right\} \subseteq\left\{\frac{\left[k^{\prime}\right]_{q^{\prime}}}{\left[r^{\prime}\right]_{q^{\prime}}}: k^{\prime} \in\left[r^{\prime}\right]\right\} .
$$

In particular, $\left[n^{\prime}\right]_{q^{\prime}}$ and $\left[r^{\prime}\right]_{q^{\prime}}$ are integer multiples of $[n]_{q}$ and $[r]_{q}$ respectively.

As the proof of this fact is quite similar to Theorem 4.2 we omit it. Unfortunately, we do not know how to prove a necessary and sufficient condition for $q$-Kneser graphs. It was shown in [4] that there is a homomorphism from $q^{m} K_{n: r}$ to $q K_{m n: m r}$, but it is not clear if these are the only homomorphisms between $q$-Kneser graphs with the same vector chromatic number.

\subsection{Hamming graphs}

In this section we focus on the graphs $H_{n, k}$ studied in Section 3.3. By Lemma 3.6 we have that $\chi_{v}\left(H_{n, k}\right)=\frac{2}{2-\frac{n}{k}}$. Consequently, $\chi_{v}\left(H_{n, k}\right)=\chi_{v}\left(H_{n^{\prime}, k^{\prime}}\right)$ if and only if $n / k=n^{\prime} / k^{\prime}$. Moreover, we have seen in Theorem 3.8 that the graph $H_{n, k}$ is UVC for any even integer $k \in[n / 2+1, n-1]$. Furthrermore, recall that the canonical vector coloring is given by $p_{x}(i)=\frac{(-1)^{x_{i}}}{\sqrt{n}}$, for all $i \in[n]$, and note that

$$
\left\langle p_{x}, p_{y}\right\rangle=\frac{n-2 d(x, y)}{n}=1-2 \frac{d(x, y)}{n},
$$

where $d(x, y)$ is the Hamming distance of two vertices of $H_{n, k}$. Next we use Lemma 4.1 to characterize homomorphisms $H_{n, k} \rightarrow H_{n^{\prime}, k^{\prime}}$, when $n / k=n^{\prime} / k^{\prime}$.

Theorem 4.4. Consider integers $n, k, n^{\prime}, k^{\prime}$ where $k<n<2 k-1, n / k=n^{\prime} / k^{\prime}$ and both $k$ and $k^{\prime}$ are even. Then, we have that $H_{n, k} \rightarrow H_{n^{\prime}, k^{\prime}}$ if and only if $n^{\prime}$ and $k^{\prime}$ are integer multiples of $n$ and $k$, respectively.

Proof. Since $n / k=n^{\prime} / k^{\prime}$ we have that $\chi_{v}\left(H_{n, k}\right)=\chi_{v}\left(H_{n^{\prime}, k^{\prime}}\right)$. By our assumptions on $n$ and $k$ it follows by Theorem 3.8 that both $H_{n, k}$ and $H_{n^{\prime}, k^{\prime}}$ are UVC. Furthermore, since $n>k$ and $n / k=n^{\prime} / k^{\prime}$, we have that $n^{\prime}>k^{\prime}$. Also, 
$n<2 k-1$ implies that $n^{\prime}<2 k^{\prime}$ and therefore $n^{\prime} \leq 2 k^{\prime}-1$. Therefore, by Lemma 4.1 and Equation (17) we get that:

$$
\left\{\frac{d}{n}: d \in[n]\right\} \subseteq\left\{\frac{d^{\prime}}{n^{\prime}}: d^{\prime} \in\left[n^{\prime}\right]\right\} .
$$

In particular, for $d=1$ this implies that there exists $d^{\prime} \in\left[n^{\prime}\right]$ such that

$$
\frac{1}{n}=\frac{d^{\prime}}{n^{\prime}},
$$

and thus $n^{\prime}=d^{\prime} n$ (and $k^{\prime}=d^{\prime} k$ ).

For the other direction, assume there exists an integer $m$ such that $n^{\prime}=m n$ and $k^{\prime}=m k$. A homomorphism $H_{n, k} \rightarrow H_{n^{\prime}, k^{\prime}}$ is given by mapping any element of $\mathbb{Z}_{2}^{n}$ to $m$ copies of that vector concatenated together.

Acknowledgements: D. E. Roberson was supported by Cambridge Quantum Computing Ltd. and the EPSRC, as well as Simone Severini and Fernando Brandao. R. Šámal was partially supported by grant GA ČR 16-19910S and by grant LL1201 ERC CZ of the Czech Ministry of Education, Youth and Sports. A. Varvitsiotis was supported in part by the Singapore National Research Foundation under NRF RF Award No. NRF-NRFF2013-13.

\section{A Canonical vector coloring of Kneser graphs}

Given integers $n$ and $r$ such that $n \geq r$, the Kneser graph $K_{n: r}$ has as vertices the $r$-subsets of $[n]$, and two are adjacent if they are disjoint. The $q$-Kneser graph $q K_{n: r}$ has the $r$-dimensional subspaces of the finite vector space $\mathbb{F}_{q}^{n}$ as its vertices, and two of these subspaces are adjacent if they are skew, i.e., if their intersection is the 0 -subspace. Note that for $n<2 r$, neither of these graphs have any edges. For $n=2 r$, the Kneser graph $K_{n: r}$ is a perfect matching, but $q K_{n: r}$ can have complicated structure. Here we only consider the case $n \geq 2 r+1$.

Both the Kneser and $q$-Kneser graphs are vertex and edge transitive, and therefore 1-walk-regular. It was shown in [8] using Theorem 2.2 that for $n \geq$ $2 r+1$, the graphs $K_{n: r}$ and $q K_{n: r}$ are both UVC. For completeness, we now give the optimal vector colorings from [8].

For $K_{n: r}$, the coordinates of the vectors in the vector coloring are indexed by $[n]$. To a subset $S \subseteq[n]$ with $|S|=r$, we assign the unit vector $p_{S} \in \mathbb{R}^{n}$ given by:

$$
p_{S}(i)= \begin{cases}\frac{r-n}{\sqrt{n r(n-r)}}, & \text { if } i \in S, \\ \frac{r}{\sqrt{n r(n-r)}}, & \text { otherwise. }\end{cases}
$$

The inner product of the vectors assigned to two $r$-subsets of $[n]$ depends only on the size of their intersection. Indeed, given two $r$-subsets $S, S^{\prime} \subseteq[n]$ with $\left|S \cap S^{\prime}\right|=k$ we have that

$$
\left\langle p_{S}, p_{S^{\prime}}\right\rangle=\frac{k}{r} \cdot \frac{n / r}{n / r-1}-\frac{1}{n / r-1},
$$

i.e., the inner product is a function of $k / r$ and $n / r$. In particular, it is minimized when $k=0$, or equivalently when $S \sim S^{\prime}$. Lastly, we show that $S \rightarrow p_{S}$ is 
an optimal vector coloring. First, note that $\chi_{v}\left(K_{n: r}\right)=n / r$. This follows by Theorem $2.2(i)$ using the fact that $K_{n: r}$ is $\left(\begin{array}{c}n-r \\ r\end{array}\right)$-regular and its least eigenvalue is $-\left(\begin{array}{c}n-r-1 \\ r-1\end{array}\right)$ (e.g., see [10, Theorem 9.4.3]). On the other hand, for $S \sim S^{\prime}$ it follows by $(19)$ that $\left\langle p_{S}, p_{S^{\prime}}\right\rangle=-\frac{1}{n / r-1}$.

The vector coloring of the $q$-Kneser graph $q K_{n: r}$ is defined analogously. Specifically, set

$$
[k]_{q}:=\frac{q^{k}-1}{q-1}=\sum_{i=0}^{k-1} q^{i},
$$

which is the number of lines contained in a $k$-dimensional subspace of $\mathbb{F}_{q}^{n}$. Then, to an $r$-dimensional subspace $S$ of $\mathbb{F}_{q}^{n}$ we assign the unit vector $p_{S} \in \mathbb{R}^{[n]_{q}}$, with entries indexed by the lines of $\mathbb{F}_{q}^{n}$, given by:

$$
p_{S}(\ell)= \begin{cases}\frac{[r]_{q}-[n]_{q}}{\sqrt{[n]_{q}[r]_{q}\left([n]_{q}-[r]_{q}\right)},}, & \text { if } \ell \subseteq S, \\ \frac{[r]_{q}}{\sqrt{[n]_{q}[r]_{q}\left([n]_{q}-[r]_{q}\right)}}, & \text { if } \ell \cap S=\{0\} .\end{cases}
$$

Lastly, note that $q K_{n: r}$ is $q^{r^{2}}\left[\begin{array}{c}n-r \\ r\end{array}\right]_{q}$ regular and its least eigenvalue is equal to $-q^{r(r-1)}\left[\begin{array}{c}n-r-1 \\ r-1\end{array}\right]_{q}$ (e.g., see $\left.[11]\right)$. Here $\left[\begin{array}{l}n \\ k\end{array}\right]_{q}$ denotes the Gaussian binomial coefficient which is equal to the number of $k$-dimensional subspaces of $\mathbb{F}_{q}^{n}$. As $q K_{n: r}$ is 1-walk-regular it follows by Theorem $2.2(i)$ that $\chi_{v}\left(q K_{n: r}\right)=[n]_{q} /[r]_{q}$. To see that $(20)$ is an optimal vector coloring note that for two $r$-dimensional subspaces with a $k$-dimensional intersection we have

$$
\left\langle p_{S}, p_{S^{\prime}}\right\rangle=\frac{[k]_{q}}{[r]_{q}} \cdot \frac{[n]_{q} /[r]_{q}}{[n]_{q} /[r]_{q}-1}-\frac{1}{[n]_{q} /[r]_{q}-1} .
$$

In particular, when $S \sim S^{\prime}$ (i.e., $k=0$ ) we get that

$$
\left\langle p_{S}, p_{S^{\prime}}\right\rangle=-\frac{1}{[n]_{q} /[r]_{q}-1} .
$$

\section{B Computations}

To compute the dimension of the span of the $\left\{p_{e}: e \in E(G)\right\}$, we may just calculate the rank of their Gram matrix. The Gram matrix of the $\left\{p_{e}: e \in\right.$ $E(G)\}$ is the matrix $M$ indexed by the edges of $G$ such that $M_{e f}=\operatorname{Tr}\left(p_{e} p_{f}\right)$. Note that the value of this trace is equal to the sum of the entries of the entrywise product of $p_{e}$ and $p_{f}$, which is the usual inner product if we were to consider $p_{e}$ and $p_{f}$ as vectors. If $e=\{i, j\} \in E(G)$ and $f=\{\ell, k\} \in E(G)$, then

$$
M_{e f}=\operatorname{Tr}\left(\left(p_{i} p_{j}^{\top}+p_{j} p_{i}^{\top}\right)\left(p_{\ell} p_{k}^{\top}+p_{k} p_{\ell}^{\top}\right)\right)=2\left(p_{j}^{\top} p_{\ell} \cdot p_{k}^{\top} p_{i}+p_{j}^{\top} p_{k} \cdot p_{\ell}^{\top} p_{i}\right) .
$$

Now, let $\tau$ be the least eigenvalue of $G$ and let $E_{\tau}$ be the projection onto its $\tau$-eigenspace. Then, recalling the definition of the canonical vector coloring, we have that $p_{j}^{\top} p_{\ell}=\left(E_{\tau}\right)_{j \ell}$, and similarly for the other inner products appearing in (22). Thus, to compute the entries of $M$, it suffices to compute the entries of $E_{\tau}$. Moreover, it suffices to compute a nonzero multiple of $E_{\tau}$ since scaling $E_{\tau}$ by $\gamma$ translates to scaling $M$ by $\gamma^{2}$, which does not affect its rank. We now describe how to do this under the assumption that $\tau$ is an integer. 
Let $\lambda_{1} \geq \ldots \geq \lambda_{n}=\tau$ be the eigenvalues of $G$ (including multiplicities) in decreasing order. Also, let $\phi$ be the characteristic polynomial of the adjacency matrix of $G$. Then $\phi$ is a monic polynomial with integer coefficients and

$$
\phi(x)=\prod_{i=1}^{n}\left(x-\lambda_{i}\right) .
$$

Also, define the polynomial $\phi_{\tau}$ as

$$
\phi_{\tau}(x)=\prod_{\lambda_{i} \neq \tau}\left(x-\lambda_{i}\right) .
$$

If $d$ is the multiplicity of $\tau$ as an eigenvalue of $G$, then

$$
\phi_{\tau}(x)=\frac{\phi(x)}{(x-\tau)^{d}} .
$$

Since $(x-\tau)^{d}$ is a factor of $\phi(x)$, if $\tau$ is an integer, then $\phi_{\tau}$ is a monic polynomial with integer coefficients.

Now let $A$ be the adjacency matrix of $G$ and consider the matrix

$$
\phi_{\tau}(A)=\prod_{\lambda_{i} \neq \tau}\left(A-\lambda_{i} I\right) .
$$

Note that all of the factors in the above product commute. If $v$ is an eigenvector of $A$ for an eigenvalue other than $\tau$, then it is easy to see that $\phi_{\tau}(A) v=0$. On the other hand, if $v$ is a $\tau$-eigenvector of $A$, then

$$
\phi_{\tau}(A) v=\left(\prod_{\lambda_{i} \neq \tau}\left(\tau-\lambda_{i}\right)\right) v \neq 0 .
$$

In other words, $\phi_{\tau}(A)$ is a nonzero multiple of $E_{\tau}$. Thus, for a 1-walk-regular graph $G$ with adjacency matrix $A$ and integer least eigenvalue $\tau$, we have the following algorithm for determining $\operatorname{dim}\left(\operatorname{span}\left\{p_{e}: e \in E(G)\right\}\right)$ :

1. Compute the characteristic polynomial $\phi$ of $A$.

2. Compute $\phi_{\tau}$ by repeatedly dividing $\phi(x)$ by $(x-\tau)$.

3. Compute $\phi_{\tau}(A)$, which is a multiple of $E_{\tau}$.

4. Use $\phi_{\tau}(A)$ and (22) to compute the Gram matrix, $M$, of the $p_{e}$.

5. Compute the rank of $M$ which is equal to $\operatorname{dim}\left(\operatorname{span}\left\{p_{e}: e \in E(G)\right\}\right)$.

Importantly, each of these steps can be done efficiently and exactly with integer arithmetic [17]. By Lemma 3.15, this algorithm allows us to determine a sufficient condition for showing a 2-walk-regular graph is a core. We note that requiring the least eigenvalue of $G$ to be integer does not seem to be very restrictive in practice.

It appears that the significant majority of the computational time is spent on determining the rank of the Gram matrix of the $p_{e}$ matrices. Based on our experience, the runtime of the algorithm appears to be roughly quadratic in the 
Table 1: Data for Strongly Regular Graphs

\begin{tabular}{|c|c|c|c|}
\hline Param. Set & Total Num. & Num. Tight & Num. Loose \\
\hline$(9,4,1,2)$ & 1 & 0 & 1 \\
\hline$(10,3,0,1)$ & 1 & 1 & 0 \\
\hline$(10,6,3,4)$ & 1 & 1 & 0 \\
\hline$(15,6,1,3)$ & 1 & 1 & 0 \\
\hline$(15,8,4,4)$ & 1 & 0 & 1 \\
\hline$(16,5,0,2)$ & 1 & 1 & 0 \\
\hline$(16,10,6,6)$ & 1 & 1 & 0 \\
\hline$(16,6,2,2)$ & 2 & 0 & 2 \\
\hline$(16,9,4,6)$ & 2 & 1 & 1 \\
\hline$(21,10,3,6)$ & 1 & 1 & 0 \\
\hline$(21,10,5,4)$ & 1 & 0 & 1 \\
\hline$(25,8,3,2)$ & 1 & 0 & 1 \\
\hline$(25,16,9,12)$ & 1 & 0 & 1 \\
\hline$(25,12,5,6)$ & 15 & 13 & 2 \\
\hline$(26,10,3,4)$ & 10 & 9 & 1 \\
\hline$(26,15,8,9)$ & 10 & 9 & 1 \\
\hline$(27,10,1,5)$ & 1 & 1 & 0 \\
\hline$(27,16,10,8)$ & 1 & 0 & 1 \\
\hline$(28,12,6,4)$ & 4 & 0 & 4 \\
\hline$(28,15,6,10)$ & 4 & 4 & 0 \\
\hline$(35,16,6,8)$ & 3854 & 2789 & 1065 \\
\hline$(35,18,9,9)$ & 3854 & 2175 & 1679 \\
\hline$(36,10,4,2)$ & 1 & 0 & 1 \\
\hline$(36,25,16,20)$ & 1 & 0 & 1 \\
\hline$(36,14,4,6)$ & 180 & 175 & 5 \\
\hline$(36,21,12,12)$ & 180 & 135 & 45 \\
\hline$(36,14,7,4)$ & 1 & 0 & 1 \\
\hline$(36,21,10,15)$ & 1 & 1 & 0 \\
\hline$(36,15,6,6)$ & 32548 & 24022 & 8526 \\
\hline$(36,20,10,12)$ & 32548 & 32536 & 12 \\
\hline$(40,12,2,4)$ & 28 & 16 & 12 \\
\hline$(40,27,18,18)$ & 28 & 17 & 11 \\
\hline$(45,12,3,3)$ & 78 & 0 & 78 \\
\hline$(45,32,22,24)$ & 78 & 77 & 1 \\
\hline$(45,16,8,4)$ & 1 & 0 & 1 \\
\hline$(45,28,15,21)$ & 1 & 1 & 0 \\
\hline$(49,12,5,2)$ & 1 & 0 & 1 \\
\hline$(49,36,25,30)$ & 1 & 0 & 1 \\
\hline$(50,7,0,1)$ & 1 & 0 & 1 \\
\hline$(50,42,35,36)$ & 1 & 1 & 0 \\
\hline$(50,21,8,9)$ & 18 & 18 & 0 \\
\hline$(50,28,15,16)$ & 18 & 17 & 1 \\
\hline$(64,18,2,6)$ & 167 & 145 & 22 \\
\hline$(64,45,32,30)$ & 167 & 0 & 167 \\
\hline
\end{tabular}


number of edges of the graph. At 20,000 edges it takes about 15 minutes for the algorithm to run in Sage [5] on our personal computers. Note that computationally testing whether a graph of this size is a core is essentially impossible.

Furthermore, we note that in [8] we presented another algorithm for determining whether a 1-walk-regular graph $G$ is uniquely vector colorable. This algorithm was based on solving a system of $|V(G)|^{2}$ linear equations in $|E(\bar{G})|$ variables. This is somewhat complementary to the algorithm given here, whose runtime depends on $|E(G)|$. In practice, the algorithm introduced above is much faster than the algorithm given in the previous work.

We now apply this algorithm to Ted Spence's list of strongly regular graphs available online [25]. In this case, we can actually compute the entries of the Gram matrix of the matrices $\left\{p_{e}: e \in E(G)\right\}$ directly from the parameters of the strongly regular graph $G$, which saves us some work. Furthermore, $G$ has integral eigenvalues unless it is a conference graph, i.e., has parameters $(4 t+$ $1,2 t, t-1, t)$ for some integer $t$. Even in this case, $G$ still has integral eigenvalues unless $4 t-1$ is not a square. Thus, considering only graphs with integral least eigenvalue is not a significant restriction for SRGs. After eliminating the graphs with non-integral eigenvalues, we were left with 73816 graphs. For each of these, we computed the Gram matrix of the matrices $\left\{p_{e}: e \in E(G)\right\}$ and then calculated its rank and compared the result to $\left(\begin{array}{c}d+1 \\ 2\end{array}\right)$, where $d$ is the multiplicity of the least eigenvalue of $G$. We call a graph tight if the rank is $\left(\begin{array}{c}d+1 \\ 2\end{array}\right)$, and loose otherwise. For each tight graph $G$, this calculation certifies that $G$ is a core. The results of these calculations are given in Table 1.

We have made some interesting observations from the data we have collected so far. There are 8526 SRGs with parameters $(36,15,6,6)$ that are not tight, but we have verified that these are all cores. The 10 graphs with parameter set $(26,10,3,4)$ are the Paulus graphs and the 10 graphs with parameter set $(26,15,8,9)$ are their complements. For each set, exactly one graph is not tight. These two graphs form a complementary pair. They correspond to the Paulus graph with the largest automorphism group (the size of this group is 120, the next largest automorphism group has size 39, the remaining Paulus graphs have less than 10 automorphisms). The 180 graphs with parameter set $(36,14,4,6)$ correspond to a class of real symmetric Hadamard matrices with constant diagonal. All but 5 of these graphs are tight. Even more striking are the 32548 graphs with parameters $(36,20,10,12)$, all but 12 of which are tight.

\section{References}

[1] László Babai. Spectra of Cayley graphs. Journal of Combinatorial Theory, Series B, 27(2):180 - 189, 1979.

[2] Andries E. Brouwer, Arjeh M. Cohen, and Arnold Neumaier. DistanceRegular Graphs. Springer, 1989.

[3] Peter J. Cameron and Priscila A. Kazanidis. Cores of symmetric graphs. Journal of the Australian Mathematical Society, 85:145-154, 102008.

[4] Ameera Chowdhury, Chris D. Godsil, and Gordon F. Royle. Colouring lines in projective space. Journal of Combinatorial Theory, Series A, pages 39$52,2006$. 
[5] The Sage Developers. Sage Mathematics Software (Version 6.9), 2015. http: //www . sagemath .org.

[6] Robert Engström, Tommy Färnqvist, Peter Jonsson, and Johan Thapper. An approximability-related parameter on graphs - properties and applications. Discrete Mathematics $\&$ Theoretical Computer Science, 17(1), 2015.

[7] Chris D. Godsil. Problems in algebraic combinatorics. The Electronic Journal of Combinatorics, 2, 1995.

[8] Chris D. Godsil, David E. Roberson, Brendan Rooney, Robert Šámal, and A. Varvitsiotis. Universal completability, least eigenvalue frameworks and vector colorings. Discrete \& Computational Geometry, 58(2):265-292, 2017.

[9] Chris D. Godsil, David E. Roberson, Robert Šámal, and Simone Severini. Sabidussi versus Hedetniemi for three variations of the chromatic number. Combinatorica, pages 1-21, 2015.

[10] Chris D. Godsil and Gordon F. Royle. Algebraic Graph Theory, volume 207. Springer GTM, 2001.

[11] Christopher D. Godsil and Karen Meagher. Erdös-Ko-Rado Theorems: Algebraic Approaches, volume 149. Cambridge University Press, 2015.

[12] Geňa Hahn and Claude Tardif. Graph homomorphisms: structure and symmetry, pages 107-166. Springer Netherlands, Dordrecht, 1997.

[13] Pavol Hell and Jaroslav Nešetřil. On the complexity of H-colourings. Journal of Combinatorial Theory, Series B, 48:92-110, 1990.

[14] Pavol Hell and Jaroslav Nešetřil. The core of a graph. Discrete Mathematics, $109(1): 117$ - 126, 1992.

[15] Pavol Hell and Jaroslav Nešetřil. Graphs and Homomorphisms. Oxford Univerity Press, 2004.

[16] David Karger, Rajeev Motwani, and Madhu Sudan. Approximate graph coloring by semidefinite programming. Journal of the ACM, 45(2):246-265, 1998.

[17] Walter Keller-Gehrig. Fast algorithms for the characteristics polynomial. Theoretical Computer Science, 36:309 - 317, 1985.

[18] Monique Laurent and Antonios Varvitsiotis. Positive semidefinite matrix completion, universal rigidity and the strong Arnold property. Linear Algebra and its Applications, 452:292-317, 2014.

[19] László Lovász. Spectra of graphs with transitive groups. Periodica Mathematica Hungarica, 6(2):191-195, 1975.

[20] Robert J. McEliece, Eugene R. Rodemich, and H.C. Rumsey. The Lovász bound and some generalizations. Journal of Combinatorics, Information 85 Systems Sciences, 3:134-152, 1978. 
[21] Jaroslav Nešetřil. Homomorphisms of derivative graphs. Discrete Mathematics, 1(3):257-268, 1971.

[22] Igor Pak and Dan Vilenchik. Constructing uniquely realizable graphs. Discrete $\& 3$ Computational Geometry, 50(4):1051-1071, 2013.

[23] David E. Roberson. Homomorphisms of strongly regular graphs. 2016. arXiv: 1601.00969

[24] Alexander Schrijver. A comparison of the Delsarte and Lovász bounds. IEEE Transactions on Information Theory, 25(4):425-429, 1979.

[25] Ted Spence's webpage: Strongly regular graphs on at most 64 vertices. http://www.maths.gla.ac.uk/ es/srgraphs.php.

[26] Saul Stahl. $n$-Tuple colorings and associated graphs. Journal of Combinatorial Theory, Series B, 20(2):185 - 203, 1976. 